\title{
Use of artificial intelligence and neural networks for analysis and gesture detection in electrical impedance tomography
}

https://doi.org/10.1515/cdbme-2020-3126

\begin{abstract}
Artificial intelligence and neural networks are getting more and more relevant for several types of application. The field of prosthesis technology currently uses electromyography for controllable prosthesis. The precision of the control suffers from the use of EMG. More precise and more collected data with the help of EIT allows a much more precise analysis and control of the prosthesis. In this paper a neural network for gesture detection using EIT is developed and presented in a user-friendly way.
\end{abstract}

Keywords: artificial intelligence, neural network, electrical impedance tomography, gesture recognition, convolutional neural network, multilayer perceptron

\section{Introduction}

In today's world, the terms artificial intelligence (AI) and neural networks (NN) have been evaluated in almost every application. The detection of gestures, performed by hand, is an application that provides a very good basis for AI. Electrical impedance tomography (EIT), which is also used in this paper, promises to be a very suitable tool for this purpose.

Detection of muscular activity for gesture recognition helps to monitor and control hand prosthesis. If a person loses a hand due to an accident, a controllable prosthesis can provide new quality of life. In this case, using AI approaches, the measured EIT data on the forearm can provide information about which gesture the person concerned is about to make.

The remainder of this paper is structured as follows: Section 2 gives the technical groundwork about AI and EIT. Section 3 describes the process of acquiring data and model selection. The two last sections 4 and 5 highlight the outcome of our research and give an overview about further work.

\footnotetext{
*Corresponding author: Christian Gibas, Medical Informatics, Am Eichenhang 50, Siegen, Germany, christian.gibas@uni-siegen.de Luca Mülln, Student of Medical Informatics, Siegen, Germany Rainer Brück, Medical Informatics, Siegen, Germany
}

\section{Knowledge About Al And EIT}

The two fields of research substantial for this implementation are Artificial Neural Networks, as a subdomain of Artificial Intelligence, and the Electrical Impedance Tomography as a medical imaging technique.

EIT is generally used to reconstruct cross-section images of encircled tissue, where different types of tissues exhibit characteristic complex resistances (impedance). By placing several electrodes directly on to the patient's skin and feeding one pair of electrodes after another with a constant supply current, it is possible to measure the voltage between the remaining pairs and thus calculate the corresponding biological impedances. In order to obtain a complete image of the enclosed tissues, this measurement pattern continues cyclically. In the most common feeding pattern, adjacent electrodes are used for power input and measurements [1]. One cycle of individual measurements represents one frame, which leads to a direct restriction in the resolution of the resulting frame by the number of electrodes used.

Although an exact definition of Artificial Intelligence has yet to be made, technologies generally can be classified as such if they show two characteristics: Fundamental similarity to human thinking or acting and independent problem solving [2]. That NN's represent both of these aspects is evident. NN are the direct and simplified mathematical abstraction of biological neural networks and generally used for complex classification problems. Although their applications are rapidly expanding to many more fields than just classification, this technique is still relevant, especially in combination with medical imaging. An artificial NN consists of many individual neurons which are connected layer by layer. Given a specified input, these neurons calculate an output based on this input and send a corresponding signal to the connected neurons of the next layer. By tweaking the weights of these connections in certain directions, the artificial NN can be optimized for a given problem. This optimization can lead to a generalization for a broader spectrum of inputs. Therefore, a data set needs to exist, where for every given input the correct corresponding output is known to train the artificial NN. The combination of artificial $\mathrm{NN}$ and EIT is relatively common for image reconstruction, while the approach of classifying raw data is fairly new $[5,6]$. 


\section{Development Of Application Based Al}

The fundamental assumption, on which this paper is based, has it's origin in the different biological impedances measured when distinct muscle groups are contracted. These differences result not only from the physiological changes during muscle contraction, but also from the different diameters of a muscle when contracting. If these measurements are characteristic, the retroactive detection of contracted muscle groups through an artificial NN is to be expected. By placing the EIT-System at an optimal reference point on the forearm, specified gestures, which use muscles with insertion points proximal of the EITSystem, are detectable.

The mobile EIT-System used in this paper contains 16 equidistand electrodes on a rubberband with $23 \mathrm{~cm}$ circumference. With the adjacent pattern, this results in 208 linear independent measurements per frame [3].

\subsection{Requirements}

The requirements of an implementation with the specified goal can be separated in certain groups. The first group of requirements is connected to the validity of the dataset which is needed for training the artificial NN. In order obtain a constant comparability for the measurements, a directive for the use of the System must be implemented. This contains an anatomical reference point for the alignment of the EIT-System, as well as the number of consecutive measurements for each gesture and the duration of one session of measuring per user. Another group of requirements is related to the underlying goal of a real-time gesture recognition, which constrict the possible usage of more electrodes for a higher resolution and the deepness and therefore complexity of the implemented artificial NN.

\subsection{Acquiring Data}

The first step in developing a gesture recognition was to acquire a large amount of training and test data while meeting all conceptual requirements. The corpus ulnae as the extension of the easily palpable olecranon was defined as the anatomical reference point. One electrode was marked and continuously used to align the System to that reference. In order to measure the most influential changes, the system was placed one third distally on the forearm, where the center of mass of many relevant muscles is located.

The defined set of gestures is split into two groups, whereas the specific gestures were chosen arbitrary. These two groups are separated by the complexity of their gestures. The first group contains basic gestures, which are characterized by simple movements and large contracting musclegroups. The second group is characterized by more complex gestures, which can involve single finger extensions or are put together by multiple basic gestures.

The default set-size, which represents the number of consecutive measurements for each gesture before proceeding to the next one, was set to ten. The System was set to measure one frame per second. These settings assure a high frequency while still guaranteeing enough distinction between consecutive measurements, so that different frames of one set comprise different information although belonging to the same gesture.

With this defined using directive of the EIT-System, 9480 individual measurements have been taken with five different users. For four of these users, one session of gestures was measured, where each session contains about 600 to 840 measurements. For a fifth user, six sessions where recorded, which are used as the foundation for training the neural network. The measurement conditions where not to be changed during a session. Readjusting or taking off the EIT-System lead to restarting the Session in order to assure direct comparability within one session.

\subsection{Model Selection}

The acquired data was used to train multiple models of artificial neural networks. One frame at a time was classified, which results in an input layer with 256 neurons, whereas for each cycle three individual values are always set to zero. Because of the image-like nature of the frames, next to multilayer perceptrons, convolutional NN where used to test the classification [4].

First, the data was validated for the usage of a neural network via a reference model of a multilayer perceptron, where the labels of the gestures where randomised. The comparison of this result, in which the detection accuracy doesn't rise significantly above chance level, with a correctly trained model assures suitable data and a basic proof of concept through a trivial procedure.

Starting with one model each for a convolutional NN and a multilayer perceptron, these models were further developed and tweaked based on their accuracy on a split validation set of measurements with a rate of 0.25 . Because all models got such a high accuracy on the split validation (above 99\%), the evaluation needed to be adjusted to a split session validation, where the $\mathrm{NN}$ was evaluated on a session of measurements disjunct from the training data. The optimal model was used for different tests and for evaluating methods to further increase the 


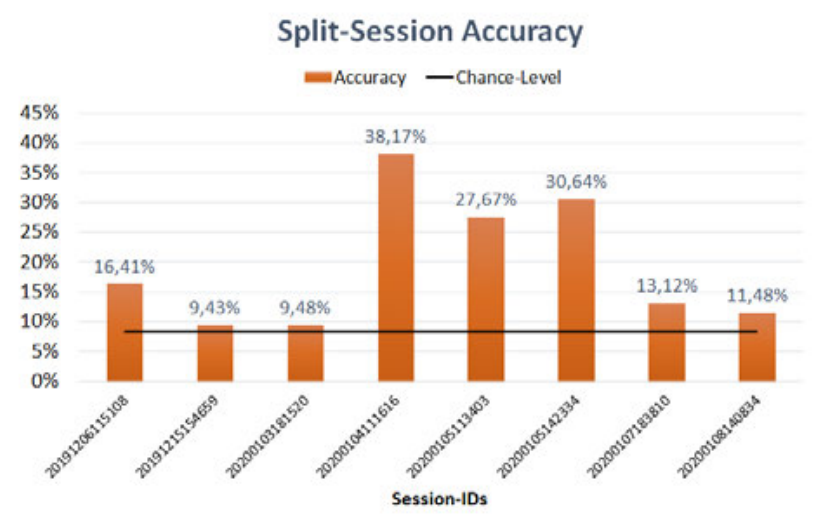

Fig. 1: Average accuracy per session over multiple runs. One run was ended through early stopping, monitoring the validation error

accuracy. These tests included cross-user validation as well as decreasing the amount of trainings data to evaluate effects on the split session validation.

\section{Results}

The validation of the data turned out positively. A quite trivial multilayer perceptron with two hidden layers, containing 128 neurons each, turned out as the optimal model for the given problem. To each hidden layer, a dropout-rate of 0.5 was applied. More complex models, especially CNN's, generally performed worse on a disjunct validation session. The inferior performance of more complex models can be attributed to the low complexity of the input data. While convolutional NN are build to base their classification on direct visual markers, like edges or vertices, the raw input data simply does not withhold such characteristics. Therefore, larger models occasionally failed to generalize completely.

Figure 1 shows the average accuracy of the chosen model on the complete set of gestures (12), where each session was separately used as validation data. Since all validation sessions were taken by the same user, the large differences in accuracy between sessions are a clear indication that small changes in the position of the system lead to major variations in the measurements. Furthermore, detection rates of up to $48 \%$ clearly indicate the basic possibility of such an implementation.

To investigate the effect of the number of training samples on the validation accuracy, these tests where repeated with a reduced training set. All sessions from different users where cut from the training data, which additionally allows to study the effect of cross-user training. This reductions resulted in a training set with about 6000 samples.
With these training conditions, all sessions which previously generalized significantly above chance level, decreased by 4-5 percentage points in accuracy. In reverse conclusion, this clearly indicates a possible increase in validation accuracy through collecting more training samples.

Additionally, the chosen gestures where individually evaluated after their average detection rate through error-matrices. Figure 2 shows the average accuracy of all gestures in descending order. The adjusted accuracy only takes those sessions into consideration, which showed significant signs of generalization.

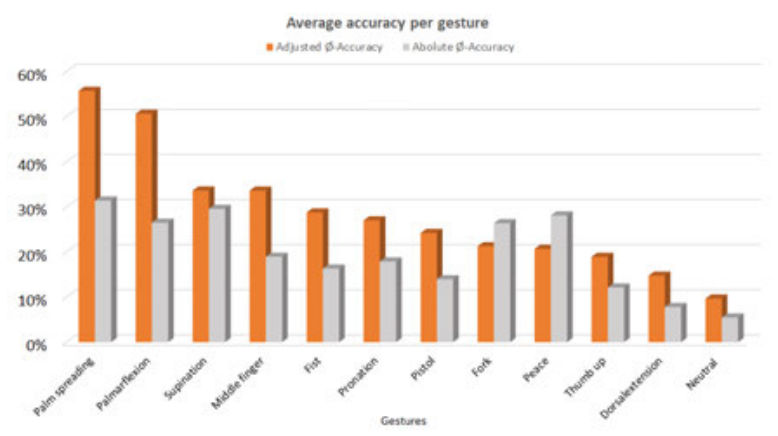

Fig. 2: Average accuracy per gesture on split session validation

\subsection{Adaptions}

To further improve the accuracy of the NN on unseen data, certain adaptions were made based on the previous test results. The gesture accuracies indicate, that certain gestures interfere with each other. Therefore, the set of gestures was reduced to the six best performing movements, considering the adjusted accuracy of each gesture.

The high accuracy of the first split validation tests indicate, that calibrating the network to the current position of the system would result in a vast increase in validation accuracy. This calibration was simulated by separating a small amount of validation data an adding these samples to the training data. With these adaptions, the implementation showed usable detection rates of up to $96 \%$ per session, as shown in Figure 3. Even cross user sessions, painted in blue in figure 3, rose to implementable accuracies. 


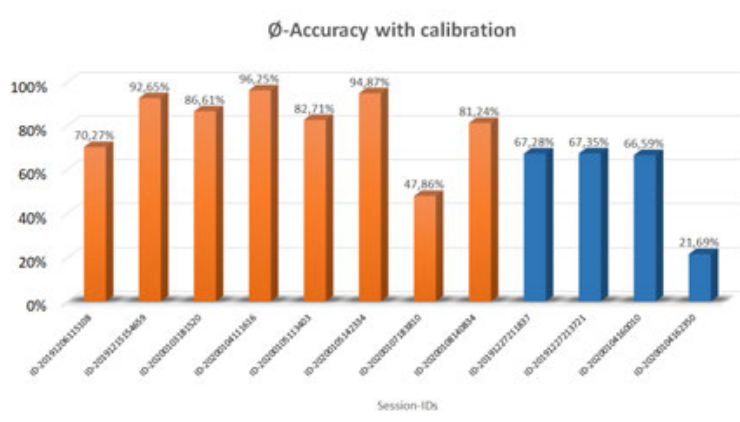

Fig. 3: Average accuracy per session with calibration

\section{$4.2 \mathrm{GUI}$}

We have developed a graphical user interface (GUI) for userfriendly operation of our work (see figure 4). The user can calibrate the EIT system initially each time it is worn with the selected gesture set. During the calibration process, images of the current gesture are displayed to the user so that the data are as similar as possible to the pre-trained neural network. Then the pre-trained neural network is adjusted with the calibrated data, so that a prediction about the current gesture can be made.

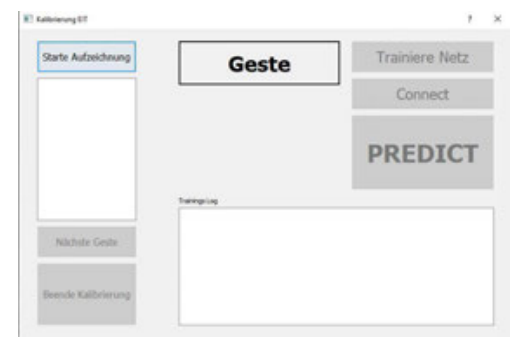

Fig. 4: GUI for realtime gesture recognition

\section{Future Work}

Our work has shown that gesture detection using AI and EIT can be realized with very high detection rates. Therefore calibration is very important for the rate. In the future, more data should be collected and the calibration should be defined even more precisely. Furthermore, the hardware has to be optimized with regard to the number of electrodes, since more electrodes should allow an even more precise classification via AI.

\section{Author Statement}

Conflict of interest: Authors state no conflict of interest. Informed consent: Informed consent has been obtained from all individuals included in this study. Ethical approval: The research related to human use complies with all the relevant national regulations, institutional policies and was performed in accordance with the tenets of the Helsinki Declaration, and has been approved by the authors' institutional review board or equivalent committee.

\section{References}

[1] Dössel O, Buzug TM. Biomedizinische Technik: Medizinische Bildgebung. 1st ed. Berlin: De Gruyter; 2014.

[2] Volker Wittpahl. Künstliche Intelligenz. Springer Vieweg; 2019.

[3] Gibas C, Grünewald A, Wunderlich H, Marx P, Brück R. A wearable EIT system for detection of muscular activity in the extremities. 2019, 41st Annual International Conference of the IEEE Engineering in Medicine and Biology Society (EMBC). 2019; pp. 2496-2499.

[4] U. Schumacher M. Schünke, E. Schulte. Hrsg. Prometheus LernAtlas-Allgemeine Anatomie und Bewegungssystem. 4: Thieme; 2014.

[5] S. J. Hamilton and A. Hauptmann, "Deep D-Bar: Real-Time Electrical Impedance Tomography Imaging With Deep Neural Networks," in IEEE Transactions on Medical Imaging, vol. 37, no. 10, pp. 2367-2377, Oct. 2018.

[6] S. Martin and C. T. M. Choi, "Nonlinear Electrical Impedance Tomography Reconstruction Using Artificial Neural Networks and Particle Swarm Optimization," in IEEE Transactions on Magnetics, vol. 52, no. 3, pp. 1-4, March 2016, Art no. 7203904 